\title{
Cytochrome-c Affects the Monoolein Polymorphism: Consequences for Stability and Loading Efficiency of Drug Delivery Systems
}

Article in Langmuir - December 2015

DOI: 10.1021/acs.langmuir.5b03507

CITATIONS

2

5 authors, including:

Leandro Ramos Souza Barbosa

University of São Paulo

60 PUBLICATIONS 433 CITATIONS

SEE PROFILE

\section{Paolo Mariani}

Università Politecnica delle Marche

214 PUBLICATIONS 3,704 CITATIONS

SEE PROFILE
READS

84

\section{Rosangela Itri}

University of São Paulo

139 PUBLICATIONS 2,119 CITATIONS

SEE PROFILE

Some of the authors of this publication are also working on these related projects:

AUTOPHAGY ACTIVATION/INHIBITION BY TRITERPENOIDS AND THE IMPACT OF MEMBRANES

INTERACTION: THERAPEUTIC IMPLICATIONS ON TUMOR RESPONSE View project 


\title{
Cytochrome-c Affects the Monoolein Polymorphism: Consequences for Stability and Loading Efficiency of Drug Delivery Systems
}

\author{
Serena Mazzoni, ${ }^{\dagger}$ Leandro R. S. Barbosa, ${ }^{\ddagger}$ Sergio S. Funari, ${ }^{\S}$ Rosangela Itri, ${ }^{\ddagger}$ and Paolo Mariani ${ }^{*}{ }^{\dagger}$ \\ ${ }^{\dagger}$ Dipartimento di Scienze della Vita e dell'Ambiente, Università Politecnica delle Marche, Ancona 60121, Italy \\ "Instituto de Fisica, Universidade de São Paulo, São Paulo 05508-020, Brazil \\ ${ }^{\S}$ HASYLAB, Hamburg D-22603, Germany
}

Supporting Information

\begin{abstract}
Structural properties and polymorphism of monoolein $(\mathrm{MO})$ in aqueous solutions have been studied for a long time, and the final picture can be considered definite. The presence of bicontinuous phases and the ability to encapsulate hydrophilic, hydrophobic, and amphiphilic compounds, together with the capability to protect and slowly release the entrapped molecules, designated MO phases as good matrices for the sustained release of drugs. Because phase stability, loading efficiency, and bioavailability are strongly correlated, the interplay between $\mathrm{MO}$ phases and entrapped compounds is worthy of investigation. In this paper, low angle

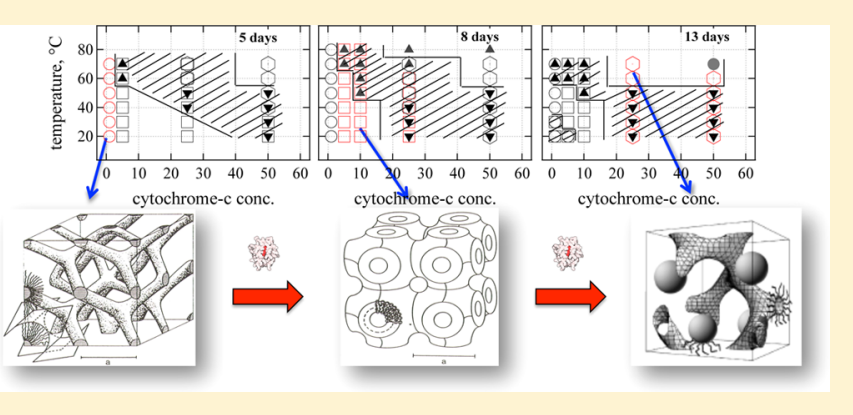
$\mathrm{X}$-ray diffraction has been used to describe the effects of a model protein (the cytochrome- $c$ ) on the monoolein cubic phases as a function of both incubation time and protein concentration in the soaking solutions. Results show that the MO polymorphism is strongly modified by the protein, underlying the very large affinity of the cytochrome-c toward monoolein. However, the different phases have a different sensibility to cytochrome-c, as phase transitions occur when the protein amount exceeds some different critical values, probably related to the structure characteristics ( 2 cytochrome-c per unit cell at the $P n 3 m$ to $\operatorname{Im} 3 m$ cubic phase transition and 10-20 cytochrome-c per unit cell at the $\operatorname{Im} 3 m$ to $\mathrm{P}_{3} 32$ cubic phase transition). Moreover, although equilibration times resulted to be quite long (more than 10 days), the fraction of cytochrome-c incorporated into the MO phases is very high (up to $20 \% \mathrm{v} / \mathrm{v}$ inside the $\mathrm{P}_{3} 32$ cubic phase). Such results are intriguing: even if they may be specific to the cytochrome-c/MO case, the need of assessing the structural characteristics of lipid matrices before their use as drug delivery systems is evident.
\end{abstract}

\section{INTRODUCTION}

Lipid-based nanosystems have been widely proposed as drug delivery systems. $^{1-4}$ Among lipid dispersions providing matrices for the sustained release of drugs, monoolein aqueous dispersions (MADs) can be mentioned. MADs are heterogeneous systems obtained by dispersion of monoolein (MO) in water and made by complex lyotropic liquid crystalline structures, such as micelles and lamellar, hexagonal, and cubic phases. The structure morphology is known to have an influence on the stability of the final product and on the delivery of encapsulated molecules, so that extensive characterization of the lipid system should be of paramount importance. ${ }^{5}$

The structural properties and polymorphism of the monoolein/water system have been studied for a long time, ${ }^{6-8}$ and the main results can be summarized considering the phase-diagram shown in Figure 1. In particular, two bicontinuous cubic phases of different space group, namely $P n 3 m$ and $I a 3 d$, dominate the temperature-concentration ( $T$ c) dependent phase diagram, while an inverse hexagonal phase is present at very high temperatures (higher than $80^{\circ} \mathrm{C}$ ), and a liquid-crystalline lamellar phase is observed at low hydration. The two cubic phases are described as infinite arrays of connected saddle surfaces with zero mean curvature in every point, ${ }^{9,10}$ the lipid monolayers being draped on each side of this surface (by definition, the G and D-IPMS surfaces for the Ia3d and the Pn $3 m$ cubic phase, respectively) and touching it with their terminal methyl groups.

Since the first extensive works, ${ }^{11-13}$ in which the phase behavior in water was clarified and the structural similarity to the physiological lipid membrane organization was found, monoolein has received great attention for applications in the pharmaceutical area. ${ }^{4,8,14}$ Indeed, the ability of encapsulation of hydrophilic, hydrophobic, and amphiphilic additives, along with the capability to protect and slowly release the entrapped molecules, makes monoolein phases excellent candidates for drug delivery systems. ${ }^{15-18}$ Moreover, MO cubic bicontinuous phases have been proved to be useful media for growing membrane protein crystals (in cubo crystallization) $)^{19-22}$ and have been suggested as organized protective media for the construction of protein biochips and affinity biosensors. ${ }^{23}$ Being strongly related to the integrity of the medium, all applications point out the special need of controlling (and eventually

Received: October 24, 2015

Revised: December 14, 2015 


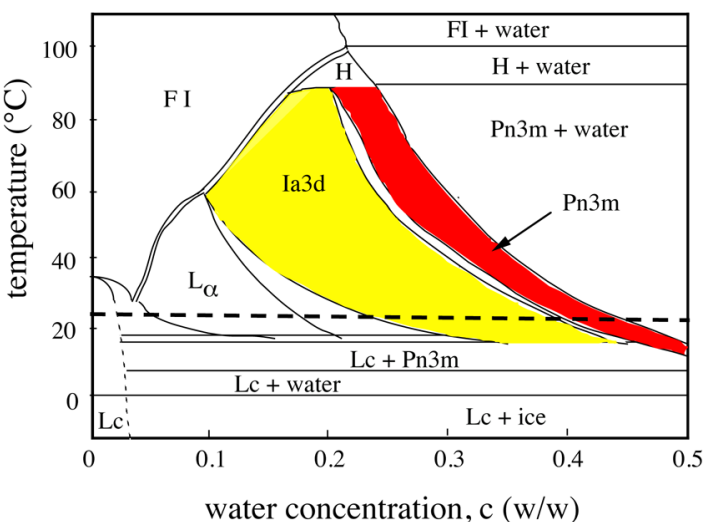

Figure 1. T-c dependent phase diagram of MO in pure water. The different phases are indicated by $H$, hexagonal phase, type II; FI, fluid isotropic; $P n 3 m$, cubic phase of space group Pn $3 \mathrm{~m}$; Ia3d, cubic phase of space group Ia3d; $L_{\alpha}$, lamellar phase, with liquid conformation of the hydrocarbon chain; $L_{c}$ lamellar crystalline phase. Regions were the assignment is ambiguous are not named. The dotted line shows the phase sequence at $25^{\circ} \mathrm{C}$.

extending) the structure and the range of stability of cubic phases. $^{3-5,24,25}$

Regarding protein incorporation, several hydrophobic or hydrophilic proteins were solubilized into MO cubic phases, resulting in different loading ability $7,13,18,21,22,26,27$ and in complicated phase diagrams. $^{7,27-29}$ For example, $18 \% \mathrm{w} / \mathrm{w}$ loading was obtained with a variety of soluble proteins (including lysozyme and myoglobin): ${ }^{26}$ results suggested the localization of protein molecules in the water medium and showed the reaching of equilibrium conditions at least within $24 \mathrm{~h}$ (but 7 days for myoglobin). Moreover, the presence of a body-centered cubic structure (suggested as Ia3d) was demonstrated in the case of lysozyme, ${ }^{26}$ even if the reported unit cell was surprisingly large. This point is relevant, because the Ia3d cubic phase has never been observed in excess water conditions.

In 1988, the ternary system composed of monoolein prepared in excess of cytochrome- $c$ water solution was considered. ${ }^{7}$ A partial $T-c$ dependent phase diagram was derived by performing X-ray diffraction experiments on samples obtained by soaking the $\mathrm{MO}$ in excess cytochrome-c/water solutions and under a progressive drying. An $\operatorname{Im} 3 m$ cubic phase, not present in the $\mathrm{MO} /$ water system, and a $\mathrm{P}_{3} 32$ cubic phase, never observed until then in lipid-containing systems (see also Funari and co-workers ${ }^{30}$ ), were detected. While the $\operatorname{Im} 3 m$ cubic phase is bicontinuous and is related to the P-IPMS surface,, 10 the structure of the $P 4_{3} 32$ is better described by reference to the skeletal graph representation of the Ia3d cubic phase. ${ }^{10}$ Indeed, the skeleton structure of the three bicontinuous cubic phases, Im $3 m, P n 3 m$, and Ia3d, can be described in terms of two 3-D networks of connected rods, mutually intertwined and unconnected. ${ }^{7}$ In the $\operatorname{Im} 3 m$, the rods are orthogonally connected 6 by 6 ; in the $P n 3 m$, the rods are joined tetrahedrally 4 by 4 ; in the Ia3d, the rods are coplanarly joined 3 by 3 . In the MO system, the cubic structures are all of type II, water-in-oil: rods are filled by the polar moiety, coated by the polar headgroups of the monoolein molecules and embedded in the

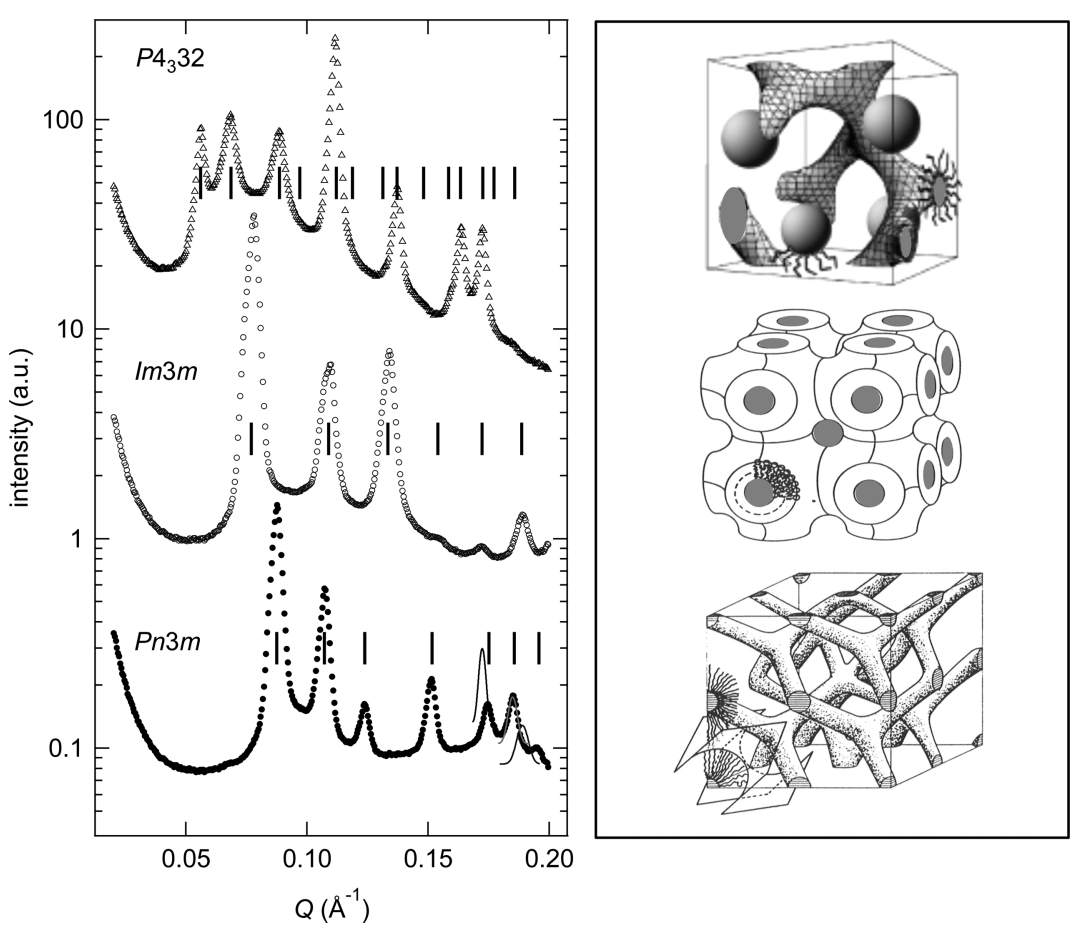

Figure 2. Cubic phases observed in monoolein in excess of cytochrome-c water solution. Left side: typical X-ray diffraction profiles observed after 8days incubation in $1 \mathrm{mg} / \mathrm{mL}$ cytochrome- $c$ solution at $25^{\circ} \mathrm{C}$ (Pn3m cubic phase, bottom), in $10 \mathrm{mg} / \mathrm{mL}$ cytochrome-c solution at $25^{\circ} \mathrm{C}(\operatorname{Im} 3 m$ cubic phase, middle) and in $50 \mathrm{mg} / \mathrm{mL}$ cytochrome-c solution at $65^{\circ} \mathrm{C}\left(\mathrm{P}_{3} 32\right.$ cubic phase, top $)$. The vertical black lines indicate the expected peak positions (from the bottom: $P n 3 m$, spacing ratios as $\sqrt{ } 2: \sqrt{ } 3: \sqrt{ } 4: \sqrt{ } 6: \sqrt{ } 8: \sqrt{ } 10: \sqrt{ } 12 ; \operatorname{Im} 3 m$, spacing ratios as $\sqrt{ } 2: \sqrt{ } 4: \sqrt{ } 6: \sqrt{ } 8: \sqrt{ } 10: \sqrt{ } 12 ; P 4{ }_{3} 32$, spacing ratios as $\sqrt{ } 2: \sqrt{ } 3: \sqrt{ } 5: \sqrt{ } 6: \sqrt{ } 8: \sqrt{9}: \sqrt{ } 11: \sqrt{ } 12: \sqrt{ } 13: \sqrt{ } 14: \sqrt{ } 16: \sqrt{ } 17: \sqrt{ } 19: \sqrt{ } 20: \sqrt{ } 21)$. Right side: sketch of the structure of the $P n 3 m$ (bottom), Im $3 m$ (middle) and $P 4_{3} 32$ (top) cubic phases. For the Pn3m, the skeleton representation of the aqueous compartments is used. The position of the IPMS D-surface is also shown; for the $\operatorname{Im} 3 \mathrm{~m}$, the representation in term of IPMS P-surface, which defines the two continuous aqueous compartments, is used. For the $P 4332$, the two different structure elements, spheres and aqueous skeleton, are represented. ${ }^{27}$ 
hydrocarbon matrix. In the $P 4_{3} 32$, one of the two 3-D networks of the Ia3d cubic phase is preserved, whereas the other is replaced by a set of identical quasi-spherical globules, each one centered on every second three-rods junction: four globules, filled by the polar moiety and surrounded by lipid molecules, are then contained in the unit cell. Accordingly, the $\mathrm{P}_{3} 32$ cubic phase is nonbicontinuous. ${ }^{10}$ The structure of $P n 3 m, \operatorname{Im} 3 m$, and $P_{3} 32$ cubic phases can be appreciated on Figure 2. The effect of cytochrome-c on MO was remarkable: ${ }^{7}$ high protein contents shift the equilibrium toward the $\operatorname{Im} 3 m$ phase, but the unit cell parameter of this phase decreases over time while the amount of protein per unit cell increases.

The same ternary system was investigated by Winter and coworkers at constant, and low, hydration level $(20 \% \mathrm{w} / \mathrm{w}$ of heavy water), varying the cytochrome-c concentration from 0.02 to $10 \% \mathrm{w} / \mathrm{w}$ and using temperature and pressure as physical parameters. ${ }^{27-29}$ X-ray diffraction and FT-IR results showed that incorporation of cytochrome- $c$ into the MO Ia3d phase affects the structure and pressure stability of the system: at high pressures, the samples with less than $0.2 \% \mathrm{w} / \mathrm{w}$ protein undergo a transition to a fluid lamellar phase, while incorporation of cytochrome-c above $0.2 \% \mathrm{w} / \mathrm{w}$ promotes the formation of the $\mathrm{P}_{3} 32$ cubic phase, whose pressure stability rises with increasing protein content. On heating, the same limiting concentration resulted crucial, as a significant influence on the lipid structure, with the probable formation of the $\mathrm{P}_{3} 32$ cubic phase, was detected above $0.2 \% \mathrm{w} / \mathrm{w}$ cytochrome- $c$. Moreover, it was observed that $\mathrm{MO} /$ cytochrome-c interactions lead to an increase in the interfacial curvature, probably accompanied by an increase of carbonyl headgroup hydration.

As a whole, results on protein incorporation in MO cubic phases suggest that the interplay between the MO-aqueous phases and the entrapped molecules is dictating phase stability and loading efficiency: if the use of cubic phases as matrices for drug delivery or as crystallization media is being pursued, it is clear that a better understanding of structural properties and phase stability is necessary. ${ }^{18}$ In the present work, we return to the $\mathrm{MO} /$ cytochrome-c/water system to investigate the effects of this protein on monoolein polymorphism in excess aqueous solutions. Two parameters have been considered, the protein concentration of the soaking solution and the incubation time. $\mathrm{X}$-ray diffraction and UV-spectroscopy experiments were performed to derive the structural properties of the phases forming in the different conditions and to calculate the amount of protein entrapped into the lipid phase. Results support the need of assessing the structural characteristics of lipid matrices for technological and pharmacological applications.

\section{MATERIALS AND METHODS}

Samples Preparation. Fully hydrated protein-containing lipid samples were prepared by soaking the MO (Sigma Chemical Co., with $>99 \%$ purity and used without further purification) in a large volume of cytochrome-c (Sigma Chemical Co., from horse heart) aqueous solutions. MO concentration was $c_{\mathrm{MO}}=25 \mathrm{mg} / \mathrm{mL}$, and the nominal concentrations of the soaking cytochrome-c solutions, $c^{*}$, were $1,5,10$, 25 , and $50 \mathrm{mg} / \mathrm{mL}$ (i.e., $0.08,0.41,0.81,2.0$, and $4.1 \mathrm{mM}$ ). After preparation, MO samples were left for days at the constant temperature of $25{ }^{\circ} \mathrm{C}$. During this time, aliquots of both the lipid phase and the supernatant were collected at different incubation time, $t$, and analyzed by X-ray diffraction (at $t$ equal 5,8 , and 13 days) and by UV-spectrophotometry ( $t$ ranging from 1 to 28 days). All experiments were repeated several times to ensure reproducibility of the data: in no case, significative differences in the structure, structural parameters and loading capacity were detected.
X-ray experiments were performed at the DESY synchrotron facility in Hamburg, Germany, on the A2 beamline. The investigated Q-range $(Q=4 \pi \sin \theta / \lambda$, where $2 \theta$ is the scattering angle and $\lambda=1.50 \AA$ the $\mathrm{X}$ ray wavelength) was $0.02-0.35 \AA^{-1}$. Experiments were performed as a function of temperature $T$, from 20 to $90{ }^{\circ} \mathrm{C}$. Scattering data were recorded on a bidimensional CCD camera of $1024 \times 1024$ pixels, radially averaged and corrected for the dark, detector efficiency and sample transmission. ${ }^{31}$ Samples were held in a vacuum tight cylindrical cell provided with thin mylar windows, which maintain the excess solution conditions. Note that thin layer chromatography tests for radiation damage showed no decomposition after diffraction experiments. In each experiment, up to 5 to 12 sharp low-angle reflections were observed. In all experimental conditions, diffraction patterns were indexed according to either 3-D cubic or 2-D hexagonal lattices: ${ }^{7,12}$ even in the coexistence regions, the indexing problem was easy to solve, because no extra peaks, which can be ascribed to the presence of unknown phases or to crystalline structures, were detected. Typical Xray diffraction profiles for the $P n 3 m, \operatorname{Im} 3 m$, and $P 4_{3} 32$ cubic phases, as observed in the current work, are shown in Figure 2: the quality of the indexing process can be easily inferred. Moreover, Figure S1 demonstrates the linear dependence between the observed and the theoretical peak positions. Once the symmetry was determined, the unit cell dimension $a$ was thus calculated. ${ }^{12}$ Errors in unit cell dimensions resulted $\pm 0.3 \AA$.

Spectroscopic measurements were performed using a Shimadzu UV3600 spectrophotometer. The absorption of each sample was measured after an appropriate dilution at $\lambda=412 \mathrm{~nm}$, and the protein concentration in the supernatant solution, $c_{\text {sol }}$, was derived from a calibration plot. Therefore, the concentration of cytochrome-c entrapped into the cubic phase, $c_{\text {in }}$ (in $\mathrm{mg} / \mathrm{mL}$ ), and the corresponding number of cytochrome-c molecules per unit volume, $n_{\text {in }}\left(\right.$ in $\left.\mathrm{ml}^{-1}\right)$, were derived by the following:

$$
\begin{gathered}
c_{\text {in }}=c^{*}-c_{\text {sol }} \\
n_{\text {in }}=c_{\text {in }} \frac{\mathcal{N}}{1000 M_{\text {cyt }}}
\end{gathered}
$$

where $M_{\text {cyt }}$ is the cytochrome-c molecular weight and $\mathcal{N}$ the Avogadro's number.

Structural Parameters and Sample Composition Determination. In a lipid phase, a number of useful molecular and structural parameters can be determined from the measured lattice parameters and sample composition. ${ }^{7,12,32}$ When the composition is unknown (as in the present case, because samples were prepared in excess solution), the phase composition can be approximatively deduced from the unit cell parameter under few hypotheses. In the present case, three basic assumptions were defined. First, sample decomposition into apolar (hydrophobic) and polar (hydrophilic) compartments was applied. ${ }^{12}$ Clearly, the hydrophilic medium included the protein. Second, the partial specific volumes of the $\mathrm{MO}$, cytochrome-c and water components (and then their molecular volumes, $\nu_{\mathrm{MO}}, \nu_{\text {cyt }}$ and $\nu_{\text {wat }}$ respectively) were considered independent of composition (as reported, for example, by Kraineva et al., Chung and Caffrey, and Pisani et al. $\left.{ }^{29,33,34}\right)$. Third, the length of the monoolein molecule, $l$, was assumed to be independent of composition and adopted phase, as already reported. ${ }^{35}$ Indeed, it has been demonstrated that in the temperature range from $0^{\circ}$ to $90^{\circ} \mathrm{C}$, the length of the $\mathrm{MO}$ molecule is well described by the equation $l=18 e^{(-0.0019 T)}$ (where $T$ and $l$ have units of ${ }^{\circ} \mathrm{C}$ and $\AA$, respectively). ${ }^{35}$

Therefore, in bicontinuous cubic phases, the volume fraction of $\mathrm{MO}, \phi_{\mathrm{MO}}$, can be calculated at any experimental condition by using the experimentally determined unit cell parameter $a$ and the calculated MO length $l$ by solving: ${ }^{36}$

$$
\phi_{\mathrm{MO}}=2 \sigma\left(\frac{l}{a}\right)+\frac{4 \pi \chi}{3}\left(\frac{l}{a}\right)^{3}
$$

where $\sigma$ and $\chi$ are parameters specific to the cubic phase (e.g., Ia3d: $\sigma$ $=3.091, \chi=-8$; Pn3m: $\sigma=1.919, \chi=-2$; Im3m: $\sigma=2.345, \chi=-4$ ). A similar equation can be also derived for the hexagonal phase. ${ }^{37}$ For 
the $P 4_{3} 32$ cubic phase, calculations can be performed by eq 2 by assuming that the unique 3-D network of $P_{4} 32$ preserves the structural properties of those observed in a Ia3d phase with similar concentration $^{10}$ and that the molecular characteristics of $\mathrm{MO}$ in both kinds of structure elements of $P_{3} 32$ are identical. Errors in $\phi_{\mathrm{MO}}$ of ca. $10 \%$ have been estimated considering the dispersion in $l$ as reported on by Briggs et al. ${ }^{35}$

According to Israelachvili, ${ }^{38}$ the self-assembly properties of lipid molecules depend on the so-called packing parameter, $\gamma$, defined by the ratio $\nu_{c} /\left(A_{c} l_{c}\right)$, where $\nu_{c}$ is the volume of the lipid hydrocarbon chain, $l_{c}$ its length and $A_{c}$ the headgroup area at the polar-apolar interface. Since $l_{\mathrm{c}}$ can be derived by solving eq 2 once determined the volume fraction of lipid chain in the unit cell (in the present case, $\phi_{\mathrm{c}, \mathrm{MO}}=\phi_{\mathrm{MO}} \nu_{\mathrm{c}, \mathrm{MO}} / \nu_{\mathrm{MO}}$, where $\nu_{\mathrm{c}, \mathrm{MO}}$ is the partial specific volume of the monoolein hydrocarbon chain, which can be calculated using the decomposition approach ${ }^{37}$ ), the packing parameter for the monoolein in a bicontinuous curved bilayer positioned on a minimal surface can be derived by the following:

$$
\gamma_{\mathrm{MO}}=\frac{\nu_{\mathrm{c}, \mathrm{MO}}}{A_{\mathrm{c}} l_{\mathrm{c}}}=\frac{1+1 / 3\langle K\rangle_{0} l_{\mathrm{c}}^{2}}{1+\langle K\rangle_{0} l_{\mathrm{c}}^{2}}
$$

In eq $3,\langle K\rangle_{0}$ is the surface-averaged Gaussian curvature on the minimal surface (note that $\langle K\rangle_{0}$ is related to the lattice parameter through the Gauss-Bonnet theorem, $\langle K\rangle_{0}=2 \pi \chi / A_{0}$, where $A_{0}$ is the area of the minimal surface within the unit cell given by $\sigma a^{2}$ ). The same eq 3 can be used to approximately derive the packing parameter in the $P_{3} 32$ cubic phase, under the assumptions reported above.

In turn, the average monoolein cross sectional area at the polarapolar interface $\left(A_{\mathrm{MO}}\right)$ can be obtained by dividing the whole surface area at the same interface, $A$, with the number of MO molecules in the unit cell. In bicontinuous cubic phases, the surface area at the polarapolar interface, integrated over one of the two monolayers within the unit cell and assumed to be parallel to the minimal surface, is given by $A=A_{0}\left(1+<K>{ }_{0} l^{2}\right)$, and then the following:

$$
A_{\mathrm{MO}}=\frac{2 A_{0}\left(1+\langle K\rangle_{0} l^{2}\right)}{a^{3} \phi_{\mathrm{MO}} / \nu_{\mathrm{MO}}}
$$

As before, under the same assumptions, this equation can be used to calculate the average monoolein cross-sectional area in the $P 4_{3} 32$ cubic phase. Errors as large as 10\% have been estimated.

Finally, information about the amount of cytochrome-c entrapped into the MO phases can be easily derived. In the cubic phases, the number of protein molecules per unit cell, $N_{\text {cyt }}$ can be calculated by the following:

$$
N_{\text {cyt }}=n_{\text {in }} \frac{a^{3} \phi_{\mathrm{MO}} / \nu_{\mathrm{MO}}}{c_{\mathrm{MO}}} \frac{1000 M_{\mathrm{MO}}}{\mathcal{N}}
$$

where $M_{\mathrm{MO}}$ is the molecular weight of the monoolein.

\section{RESULTS AND DISCUSSION}

Experimental Results. X-ray diffraction experiments were performed on MO samples prepared in excess of cytochrome-c solutions. By way of illustration, Figure 3 displays the diffraction profiles obtained as a function of temperature from MO samples prepared in different soaking solutions after 8 days of incubation. The changes in peak positions and intensities show that the liquid-crystal phases are concentration and temperature-dependent. Similar results have been obtained from samples at different incubation times (see Figure S2).

The analysis of the spacing ratios of the diffraction peaks allows the identification of the different phases and the construction of the phase diagram (see Figure S1). As an example, the spacing ratios of the diffraction peaks observed for the $1 \mathrm{mg} / \mathrm{mL}$ sample of Figure 3 scale as $\sqrt{2}: \sqrt{3}: \sqrt{4} \ldots$ and indicate the presence of the $P n 3 m$ cubic phase, while the peak
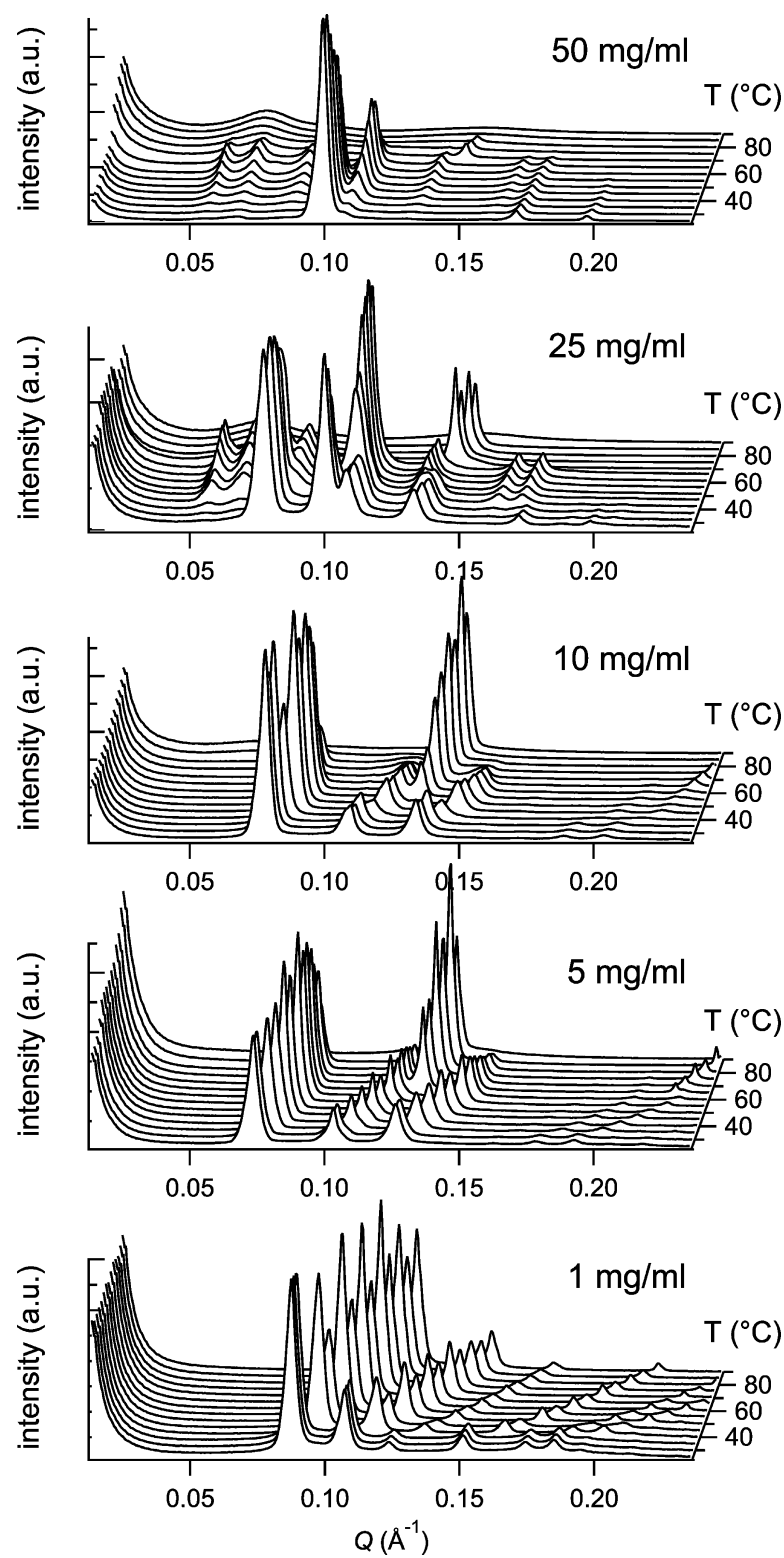

Figure 3. X-ray diffraction profiles from $\mathrm{MO}$ samples incubated for 8 days in different soaking solutions. Each set of curves corresponds to a different cytochrome-c concentration, $c^{*}$, as indicated in the right. In each set, curves correspond to different temperatures, from 20 to 90 ${ }^{\circ} \mathrm{C}$, as indicated by the scale in the right axis.

spacing ratios in the order as $\sqrt{ } 2: \sqrt{ } 4: \sqrt{ } 6: \ldots$ observed in the low temperature diffraction profiles of the 5 and $10 \mathrm{mg} / \mathrm{mL}$ samples indicate the occurrence of the $\operatorname{Im} 3 m$ cubic phase (Figure 2).

The $T-c^{*}$ dependent phase diagrams, derived at the different incubation times, are thus shown in Figure 4. It can be observed that the $P n 3 m$ cubic phase, which exists in pure monoolein in excess of water up to about $90{ }^{\circ} \mathrm{C}$ (see Figure 1), is destabilized by the presence of cytochrome- $c$, which induces the formation of the $\operatorname{Im} 3 \mathrm{~m}$ cubic phase and the appearance of the hexagonal phase at temperature lower than $90^{\circ} \mathrm{C}$. The $\operatorname{Im} 3 m$ cubic phase occupies the central part of the phase diagrams, becoming the favorite structure forming in excess cytochrome-c solutions when the incubation time is sufficiently long and $c^{*}$ is sufficiently high. However, in such a condition, another cubic phase extends on a large area on the phase diagram, the 

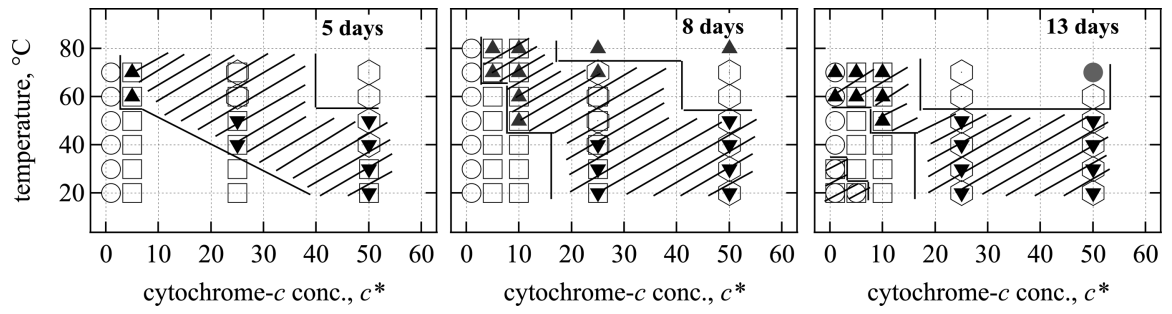

Figure 4. Phase diagrams for MO samples obtained at different incubation time. From the left: 5, 8, and 13 days. The diagram axes are the temperature of the X-ray diffraction experiment (left axis) and the cytocrome-c concentration of the soaking solution (bottom axis). The different observed phases are indicated by different symbols: $O, P n 3 m$ cubic; $\square, \operatorname{Im} 3 m$ cubic; $\bigcirc, P 4_{3} 32$ cubic; $\boldsymbol{\Delta}, H$ hexagonal; $\nabla$, Hex* reentering hexagonal. The gray dot indicates the presence of a micellar phase. Biphasic regions are sketched by lines.

nonbicontinuous $\mathrm{P}_{3} 32$ cubic phase. Further increasing the concentration of the soaking solutions, a second hexagonal phase, indicated by Hex*, is found to be present. This phase exists at room temperature and extend up to 40 or $50{ }^{\circ} \mathrm{C}$, depending on incubation time. The presence of a hexagonal phase in this region of the phase diagram is very unexpected: accordingly, we will show that its structural features are different from those derived for the hexagonal phase forming at high temperature.

The reported results have been discussed considering the nominal concentration of the soaking solutions, but it is evident that they depend on the amount of cytochrome-c entrapped into the cubic phase. Figure 5 shows the incubation-time
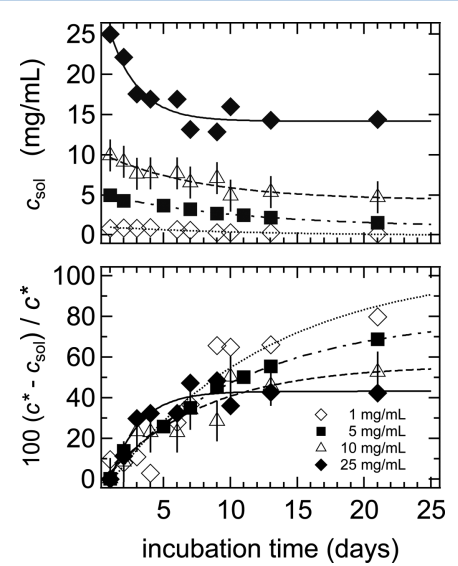

Figure 5. Dependence of the cytochrome-c concentration on incubation time. Upper frame: protein concentration in the supernatant solution, $c_{\text {sol }}$. Lines are exponential fits to the data and the corresponding decay constant $\tau$ are 11.8, 10.6, 7.3, and 2.1 days for the $1,5,10$, and $25 \mathrm{mg} / \mathrm{mL}$ cytochrome- $c$ soaking solutions, respectively. Lower frame: percentage of cytochrome-c entered into the MO phase. Lines are eye guides. For the sake of clarity, error bars are shown only in one case.

dependence of the protein concentration inside the MO phases, as obtained by spectroscopy measurements. Two comments are in order. First, at all protein concentrations, more than 10 days are necessary to establish equilibrium conditions, e.g., to observe a constant cytochrome- $c$ concentration in the supernatant solutions. The equilibration time is shorter for more concentrated soaking solutions (see the upper frame), supporting the idea of a diffusion process down the concentration gradient. Note that low-concentrated cythocrome-c samples did not reach equilibrium conditions in the considered time. Indeed, the concentration gradient affects the rate of diffusion: the incubation-time decay constant $\tau$ changes continuously from 12 to 2 days when the concentration of the soaking solution changes from 1 to $25 \mathrm{mg} / \mathrm{mL}$, respectively. Second, the fraction of protein incorporated into the MO phase can be very high, as clearly observed when the nominal concentration of the soaking solution is low. When incubated in a $1 \mathrm{mg} / \mathrm{mL}$ solution, the cytochrome- $c$ entered into the lipid phase is more than $80 \%$ of total cytochrome dissolved in the soaking solution (see the lower frame). This result demonstrates a very large affinity of the protein toward the monoolein phase, as previously suggested. ${ }^{27,29}$

Structural Parameters. Unit cell dimensions, determined from X-ray diffraction profiles as previously discussed, and structural parameters determined using eqs 2 and 4, are reported as a function of temperature and composition of the bathing solutions in Figure 6 for samples incubated 8 days. In the SI, unit cell dimensions for the other incubation times are reported (Figure S3).

It appears evident that the loading of cytochrome-c in the MO phases modifies not only the polymorphism (with the appearance of nonbicontinuous phases), but also the unit cell parameter of an equivalent phase forming in different experimental conditions. For example (see Table S1), at 25 ${ }^{\circ} \mathrm{C}$, the unit cell parameter of the $\operatorname{Im} 3 m$ phase forming in the soaking solution at $c^{*}=5 \mathrm{mg} / \mathrm{mL}$ is $121.7 \AA$ if measured over 8 days of incubation and $118.5 \AA$ if measured over 13 days. Moreover, the $\operatorname{Im} 3 m$ unit cell reduces to $114.8 \AA$ if measured after 8 days of incubation at $c^{*}=10 \mathrm{mg} / \mathrm{mL}$. According to previous results, ${ }^{7}$ the entering of cytochrome-c into the cubic phases produces a shrinking of the cell. Moreover, the unit cell parameter for all the observed phases continuously decreases on heating (see Figure 6), again in agreement with the thermally induced dehydration previously reported for monoolein in pure water. ${ }^{7,27,39}$ Because of the two effects, the extent of unit cell reduction differs for the distinct phases, and it results smaller when the amount of cytochrome- $c$ in the soaking solutions was larger (see also Figure S3).

The consequence of the observed dependence is dual: at one side, a continuous reduction of the thermal dehydration coefficient $\left(\mathrm{d} \phi_{\mathrm{MO}} / \mathrm{d} T\right.$, e.g. the slope of the curve obtained by linear fitting $\phi_{\mathrm{MO}}$ data as a function of temperature, see the second column of Figure 6) is detected with the increase of the cytochrome- $c$ concentration. Noticeable is the fact that $\mathrm{d} \phi_{\mathrm{MO}} /$ $\mathrm{d} T$ becomes negative at the largest investigated protein concentrations, indicating that in these conditions dehydration is no more effective. On the other side, the monoolein cross sectional area at the polar-apolar interface $A_{\mathrm{MO}}$ is observed to increase as a function of temperature (see the last column in the same Figure). According to $\phi_{\mathrm{MO}}$ data, the enlargement is more evident when monoolein has been incubated with 

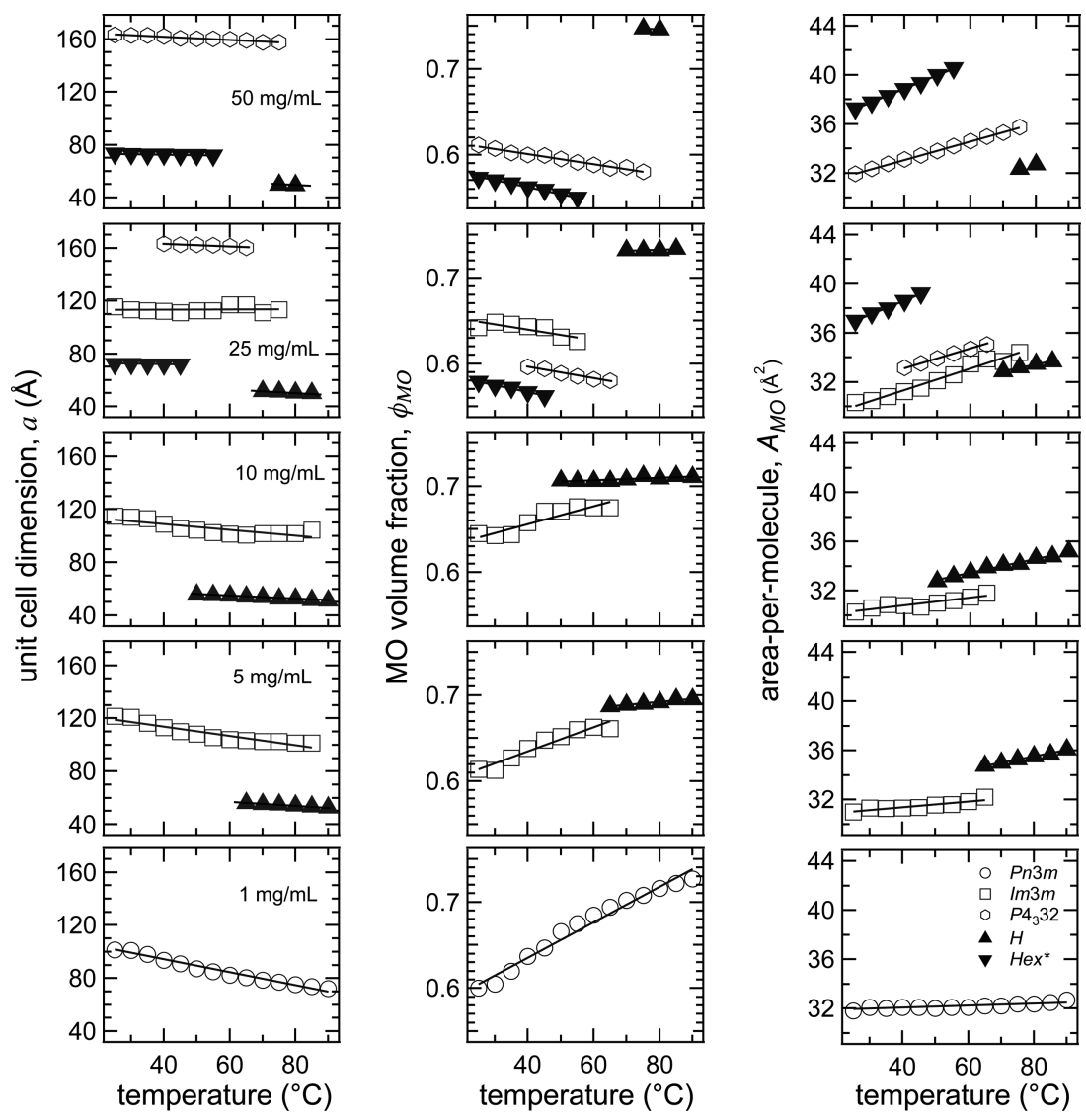

Figure 6. Temperature dependence of the structural parameters for the MO phases obtained after 8 days of incubation in different soaking solutions. Cytochrome- $c$ concentrations are indicated in the left frames, while symbols are explained in the right, bottom frame. Columns from the left: unit cell $(a, \AA)$, monoolein volume fraction $\left(\phi_{\mathrm{MO}}\right)$ and monoolein cross sectional area at the polar-apolar interface $\left(A_{\mathrm{MO}}\right)$. Lines are linear fit to the data.

solutions containing large amount of cytochrome-c. The two thermal effects are related, but they suggest that the monoolein shape is strongly dependent on the amount of entrapped protein: the inverted truncated cone shape of $\mathrm{MO}$ tends to become more cylindrical at high temperature, an effect favored by the presence of cytochrome-c.

The last point concerns the two hexagonal phases. Figure 6 shows that their unit cell parameters (which in this phase correspond to the distance between the closest rod-like structure elements) are very different. Considering the samples incubated for 8 days (Figure 6), the unit cell of the $H$ hexagonal phase at $80{ }^{\circ} \mathrm{C}$ continuously varies from 54.1 to $49.4 \AA$ when the cytochrome-c concentration of the soaking solutions changes from 5 to $50 \mathrm{mg} / \mathrm{mL}$, respectively. These values compare well with the $50 \AA$ unit cell observed in the $\mathrm{MO} /$ water system at $90{ }^{\circ} \mathrm{C}$ in fully hydrated conditions. ${ }^{39} \mathrm{On}$ the contrary, the hexagonal Hex* phase only exists below $60{ }^{\circ} \mathrm{C}$ and is characterized by a unit cell rather constant and as larger as $72 \AA$. Such a unit cell can only be explained by an unusually large cross sectional area of the $\mathrm{MO}$, favored by the presence of cytochrome-c (Figure 6 shows indeed that $A_{\mathrm{MO}}$ in $\mathrm{Hex}^{*}$ is very different from the rather similar values observed in the other phases; note also that $\phi_{\mathrm{MO}}<0.6$, indicating a corresponding massive hydration of this phase). According to the changes in $\mathrm{d} \phi_{\mathrm{MO}} / \mathrm{d} T$ and in the MO shape, it can be suggested that Hex* is a reentrant metastable phase.

Loading Efficiency. According to eq 5, the number of cytochrome-c molecules per unit cell has been calculated from sample composition (note that in the 2-phase coexistence regions, the MO-to-cytochrome- $c$ mol ratio has been assumed to be the same in both phases). Results obtained at $25{ }^{\circ} \mathrm{C}$ are reported in Figure 7.

Interestingly, the whole results point out that $\mathrm{MO}$ phases are able to accommodate a very large number of cytochrome- $c$ molecules (up to 60 protein molecules per unit cell in the $\mathrm{P}_{3} 32$ cubic phase). The loading kinetics is also clear: in bicontinuous cubic phases, the loading is linearly dependent on incubation time, and independent of the formed phase. On the contrary, a large discontinuity is observed between the bicontinuous and nonbicontinuous cubic phases: the $\mathrm{P}_{3} 32$ cubic phase is indeed very rich in cytochrome-c, and a further protein diffusion seems inhibited. In turn, the loading rate $\left(\mathrm{d} N_{\text {cyt }} / \mathrm{d} t\right)$ for bicontinuous cubic phases shows a linear dependence on the concentration of the soaking solutions, while for the $P 4_{3} 32$ cubic phase is quite constant (Figure 7, lower frame).

These data are maybe not useful to compare the loading capacity of the different MO phases, as unit cells are rather different. Therefore, the volume fraction of cytochrome-c in the cubic samples has been calculated. Results are reported in Figure 8 as a function of the nominal concentration of cytochrome- $c$ in the soaking solutions. In the investigated range, the protein volume fraction in the entire sample could be as high as $25 \%$ (i.e., $28 \mathrm{mM}$ ). Two aspects should be noticed. First, the loading variations reported as volume fraction are irrespective of phase transitions, even when the transition boundaries from bicontinuous to nonbicontinuous phases are crossed. Second, the cytochrome-c molar concentration in the 

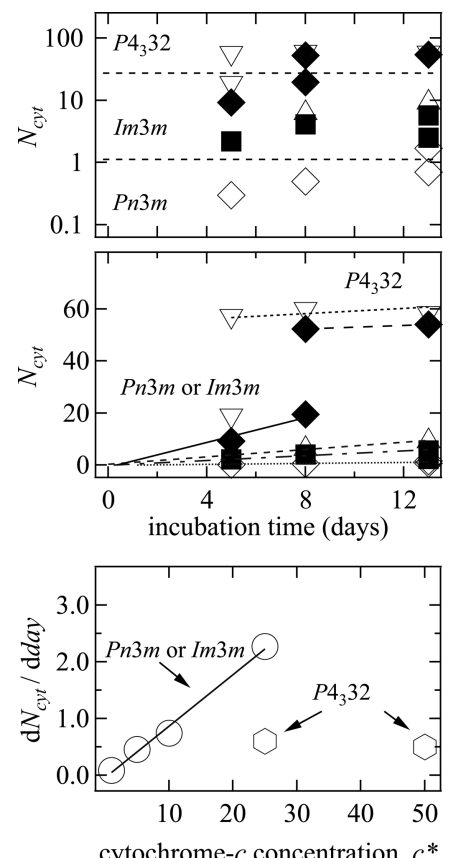

Figure 7. Amount of cytochrome- $c$ entrapped in the $\mathrm{MO}$ phases at 25 ${ }^{\circ} \mathrm{C}$. Note that the $\mathrm{P}_{3} 32$ points at 5 days of incubation at $50 \mathrm{mg} / \mathrm{mL}$ and at 8 days of incubation at $25 \mathrm{mg} / \mathrm{mL}$ have been measured at 40 ${ }^{\circ} \mathrm{C}$. Upper frames: dependence of the number of cytochrome-c molecules per unit cell, $N_{\text {cyt }}$, on the incubation time. The different symbols indicate the nominal concentration of the soaking solutions: $\diamond 1 \mathrm{mg} / \mathrm{mL}, \square 5 \mathrm{mg} / \mathrm{mL}, \triangle 8 \mathrm{mg} / \mathrm{mL}, 25 \mathrm{mg} / \mathrm{mL}$, and $\nabla 50 \mathrm{mg} /$ $\mathrm{mL}$. Lines are linear fitting to the experimental data. The top frame emphasizes the $t$-dependence by using a log scale for $N_{\text {cyt }}$ : horizontal dotted lines indicate the $N_{\text {cyt }}$ values at the two cubic-to-cubic phase transitions. Lower frame: Dependence on the concentration of the soaking solution of the slope of the fitting lines shown in the upper frame.

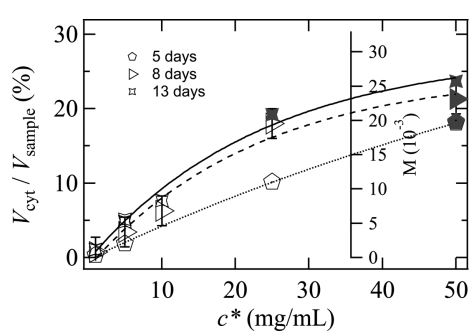

Figure 8. Volume fraction of cytochrome-c in cubic samples at $25^{\circ} \mathrm{C}$. Lines, whose style refers to the different incubation times, are quadratic fit to the $V_{\text {cyt }} / V_{\text {sample }}$ data. Open and closed symbols refer to the two bicontinuous cubic phases and to the $P 4_{3} 32$ cubic phase, respectively. The second vertical scale indicates the molar concentration of the cytochrome- $c$ in the cubic sample, calculated considering the total cubic sample volume.

MO sample is from 5 to 15 times larger than the nominal soaking solution, suggesting that the cubic phases behave like an efficient sponge ables to concentrate the protein (the cytochrome-c molar concentration, calculated considering only the aqueous compartment inside the cubic phase, attains the 60 $\mathrm{mM}$ in the best case, e.g., $P 4_{3} 32$ cubic phase after 13 days of incubation in the $50 \mathrm{mg} / \mathrm{mL}$ soaking solution, i.e., in a $4.1 \mathrm{mM}$ cytochrome-c solution).

Data in Figure 7 also indicate that the different monoolein phases have a different sensibility to cytochrome-c, as phase transitions occur when the protein amount exceeds some critical values (see the top frame). In particular, a transition from $P n 3 m$ to $\operatorname{Im} 3 m$ phase is observed when more than 1 cytochrome- $c$ enters the unit cell. As already discussed, this critical value can be reached in a rather short time, when the protein concentration of the soaking solution is high, or after a long incubation time, when the concentration of the soaking solution is low. However, a second transition from $\operatorname{Im} 3 m$ to $P_{3} 32$ cubic phase is detected when the number of cytochromec per unit cell is larger than 10 . It should be clear that these $N_{\text {cyt }}$ threshold values should be considered only indicative, because wide biphasic regions characterize the phase transition boundaries.

In any case, such numbers are sound, especially if the skeleton representation of cubic phases is considered (Figure 2): the $P n 3 m$ cubic cell contains 4 rods, joined tetrahedrally 4 by 4. According to the Crystallographic Tables, ${ }^{40}$ the rods occupy positions $e$, of symmetry $3 m$, and their junction the position $a$, of symmetry $\overline{4} 3 \mathrm{~m}$. One molecule of cytochrome-c can be accommodated at the most in this phase, probably sitting at the junction position. In the $\operatorname{Im} 3 m$ unit cell, there are 12 half-rods, cubically joined 6 by 6 which occupy positions $e$, of symmetry $4 \mathrm{~mm}$, and 2 junctions, which occupy positions $a$, of symmetry $m \overline{3} \mathrm{~m}$. Therefore, the formation of two junctions could be the way to easily load 2 cytochrome-c molecules per cell. Continuing incubation, the $\operatorname{Im} 3 m$ appears able to accommodate a huge number of protein molecules (over 13 days of incubation in the $10 \mathrm{mg} / \mathrm{mL}$ soaking solution, about 10 molecules of cytochrome-c are loaded per unit cell into the $\operatorname{Im} 3 m$ cubic phase, so the corresponding cytochrome- $c$ molar concentration calculated considering only the aqueous compartment inside the $\operatorname{Im} 3 m$ cubic phase is $22 \mathrm{mM}$, but the $\mathrm{P}_{3} 32$ has more special position to load the cytochrome-c. In this phase, the unit cell contains a network of 12 rods joined 3 by 3 in 4 junctions, which occupy general positions, plus 4 globules at positions $a$ of symmetry $32,{ }^{40}$ i.e., 8 hydrophilic large sites where proteins can be confined. Note that the $\operatorname{Im} 3 m$ to $P 4_{3} 32$ phase transition occurs just when more than 10 molecules of cytochrome- $c$ should be loaded per unit cell.

Cytochrome-c and MO Phase Transitions. The last point to be discussed concerns the $\mathrm{MO} /$ cytochrome-c possible interactions and the origin of the phase transitions. Figure 9 reports the $\mathrm{MO}$ packing parameter, calculated as indicated by eq 3 , as a function of the number of cytochrome-c per unit cell. In this master plot, all the samples in cubic phase have been associated together (independent of soaking solution concentration or incubation time) and only the kind of cubic phase has been highlighted. The behavior is very interesting: within each phase region, the packing parameter of monoolein increases with cytochrome-c loading, confirming that the entering of the protein induces a continuous dehydration of the polar/apolar interface, but the sawtooth nature of the graph implies that there is a limiting $\gamma_{\mathrm{MO}}$ at which the phase transition occurs. Indeed, the change from the Pn $3 m$ cubic phase, where 1 cytochrome-c molecule can be easily confined, to the $\operatorname{Im} 3 m$ cubic phase, where 2 protein molecules are loaded, and then to the $P 4_{3} 32$, where more than $10-20$ proteins can be definitely confined, should be considered an attempt by the MO to preserve the best packing parameter. Noticeable, the same effect on the packing parameter has been observed on $\mathrm{MO}$ / water system at the $P n 3 m$ to Ia3d cubic to cubic phase transition at $25{ }^{\circ} \mathrm{C}$ : the phase transition is associated with a redistribution of structural characteristics (e.g., curvature of the 

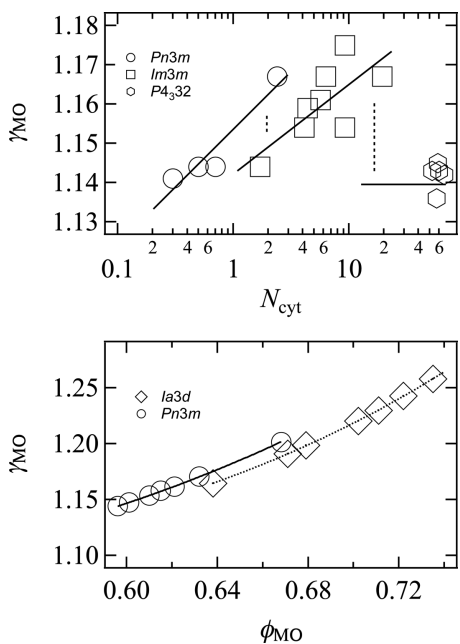

Figure 9. Dependence of the MO packing parameter on the number of cytochrome-c molecules per unit cell at $25{ }^{\circ} \mathrm{C} . \gamma_{\mathrm{MO}}$ has been calculated using eq 3 . Note the discontinuities in correspondence to the phase transitions. The reported lines are guides to the eyes.

polar-apolar interface and area-per molecule) that consent to recover the initial, spontaneous molecular packing parameter (or at least to reduce its value).

\section{CONCLUSIONS}

In this paper, the $\mathrm{MO} /$ cytochrome-c/water system has been used as a model system to analyze stability and loading characteristic of cubic phases. Two parameters have been considered, e.g., protein concentration of the soaking solution used to prepare the samples and incubation time. The calculated amount of protein entrapped into the lipid phase and the structural properties of the MO phases forming in the different conditions show the very large affinity of the cytochrome-c toward the monoolein phases, which in contrast provide a good matrix to load the protein. In the final conditions, the cytochrome-c molar concentration in the MO sample is from 5 to 15 times larger than the nominal soaking solution and in the best case the protein molar concentration in-cubo, calculated considering only the aqueous compartment inside the cubic phase, attains the $60 \mathrm{mM}$ when the soaking solution is less than $4 \mathrm{mM}$.

However, the cytochrome-c induces large structural rearrangements in the MO phases: cubic-to-cubic phase transitions appear related to the need of increasing the number of crystallographic sites (or to provide larger hydrophilic sites) where proteins can be confined and to the attempt of monoolein to recover the unstressed, initial packing parameter, or at least to reduce its value. The sequence $P n 3 m, \operatorname{Im} 3 m$, and $P_{4} 32$ appears to fulfill both conditions. Probably, more complex effects should be listed, especially if the presence of the large biphasic regions, the occurrence of two different hexagonal phases (one of which extremely hydrated) and the limit of the applied hypothesis are properly considered.

In any case, results support the need of assessing the structural characteristics of lipid matrices for technological or pharmacological applications of lipidic systems.

\section{ASSOCIATED CONTENT}

S Supporting Information

The Supporting Information is available free of charge on the ACS Publications website at DOI: 10.1021/acs.langmuir.5b03507.

$\mathrm{X}$-ray diffraction results for samples equilibrated 5 and 13 days. Structural data at $25{ }^{\circ} \mathrm{C}(\mathrm{PDF})$

\section{AUTHOR INFORMATION}

\section{Corresponding Author}

*E-mail: mariani@univpm.it.

\section{Notes}

The authors declare no competing financial interest.

\section{ACKNOWLEDGMENTS}

This work was supported by grant 401252/2014-0, Conselho Nacional de Pesquisa (CNPq). We gratefully acknowledge Università Politecnica delle Marche for the Visiting Professor Fellowship to R.I. R.I. and L.B. also acknowledge CNPq for Research fellowships.

\section{REFERENCES}

(1) Bunjes, H.; Drechsler, M.; Koch, M. H.; Westesen, K. Incorporation of the model drug ubidecarenone into solid lipid nanoparticles. Pharm. Res. 2001, 18, 287-293.

(2) Esposito, E.; Eblovi, N.; Rasi, S.; Drechsler, M.; Di Gregorio, G. M.; Menegatti, E.; Cortesi, R. Lipid-based supramolecular systems for topical application: a preformulatory study. AAPS PharmSci 2003, 5, $30-47$.

(3) Shah, J. C.; Sadhale, Y.; Chilukuri, D. M. Cubic phase gels as drug delivery systems. Adv. Drug Delivery Rev. 2001, 47, 229-250.

(4) Conn, C. E.; Drummond, C. J. Nanostructured Bicontinuous Cubic Lipid Self-Assembly Materials as Matrices for Protein Encapsulation. Soft Matter 2013, 9, 3449-3464.

(5) Bunjes, H.; Unruh, T. Characterization of lipid nanoparticles by differential scanning calorimetry, X-ray and neutron scattering. Adv. Drug Delivery Rev. 2007, 59, 379-402.

(6) Longley, W.; McIntosh, T. A bicontinuous tetrahedral structure in a liquid crystalline lipid. Nature 1983, 303, 612-614.

(7) Mariani, P.; Luzzati, V.; Delacroix, H. Cubic phases of lipidcontaining systems. Structure analysis and biological implications. J. Mol. Biol. 1988, 204, 165-189.

(8) Kulkarni, C. V.; Wachter, W.; Iglesias-Salto, G.; Engelskirchen, S.; Ahualli, S. Monoolein: a magic lipid ? Phys. Chem. Chem. Phys. 2011, 13, 3004-3021.

(9) Scriven, L. E. Equilibrium bicontinuous structure. Nature 1976, $263,123-125$.

(10) Luzzati, V.; Vargas, R; Mariani, P.; Gulik, A.; Delacroix, H. Cubic phases of lipid-containing systems. Elements of a theory and biological connotations. J. Mol. Biol. 1993, 229, 540-551.

(11) Gulik-Krzywicki, T.; Shechter, E.; Luzzati, V.; Faure, M. Interactions of Proteins and Lipids: Structure and Polymorphism of Protein - Lipid - Water Phases. Nature 1969, 223, 1116-1121.

(12) Luzzati, V. In Biological Membranes; Chapman, D., Ed.; Academic Press: London, 1968; pp 71-123.

(13) Larsson, K.; Lindblom, G. Molecular amphiphile bilayers forming a cubic phase in amphiphile-water systems. J. Dispersion Sci. Technol. 1982, 3, 61-66.

(14) Angelova, A.; Angelov, B.; Papahadjopoulos-Sternberg, B.; Ollivon, M.; Bourgaux, C. Proteocubosomes: nanoporous vehicles with tertiary organized fluid interfaces. Langmuir 2005, 21, 41384143.

(15) Esposito, E.; Drechsler, M.; Mariani, P.; Sivieri, E.; Bozzini, R.; Montesi, L.; Menegatti, E.; Cortesi, R. Nanosystems for skin hydration: a comparative study. Int. J. Cosmet. Sci. 2007, 29, 39-47. 
(16) Esposito, E.; Mariani, P.; Ravani, L.; Contado, C.; Volta, M.; Bido, S.; Drechsler, M.; Mazzoni, S.; Menegatti, E.; Morari, M.; Cortesi, R. Nanoparticulate lipid dispersions for bromocriptine delivery: Characterization and in vivo study. Eur. J. Pharm. Biopharm. 2012, 80, 306-314.

(17) Conn, C. E.; Mulet, X.; Moghaddam, M. J.; Darmanin, C.; Waddington, L. J.; Sagnella, S. M.; Kirby, N.; Varghese, J. N.; Drummond, C. J. Enhanced uptake of an integral membrane protein, the dopamine D2L receptor, by cubic nanostructured lipid nanoparticles doped with $\mathrm{Ni}(\mathrm{II})$ chelated EDTA amphiphiles. Soft Matter 2011, 7, 567-578.

(18) Van't Hag, L.; Shen, H. H.; Lu, J.; Hawley, A. M.; Gras, S. L.; Drummond, C. J.; Conn, C. E. Deconvoluting the effect of the hydrophilic and hydrophobic domains of an Amphiphilic Integral Membrane Protein in Lipid Bicontinuous Cubic Mesophases. Langmuir 2015, 31, 12025-12034.

(19) Chiu, M. L.; Nollert, P.; Loewen, M. C.; Belrhali, H.; PebayPeyroula, E.; Rosenbusch, J. P.; Landau, E. M. Crystallization in cubo: general applicability to membrane proteins. Acta Crystallogr., Sect. D: Biol. Crystallogr. 2000, 56, 781-784.

(20) Wallace, E.; Dranow, D.; Laible, P. D.; Christensen, J.; Nollert, P. Monoolein Lipid Phases as Incorporation and Enrichment Materials for Membrane Protein Crystallization. PLoS One 2011, 6, e24488.

(21) Liang, Y.-L.; Conn, C. E.; Drummond, C. J.; Darmanin, C. Uptake of the butyrate receptors, GPR41 and GPR43, in lipidic bicontinuous cubic phases suitable for in meso crystallization. J. Colloid Interface Sci. 2015, 441, 78-84.

(22) Conn, C. E.; Darmanin, C.; Sagnella, S. M.; Mulet, X.; Greaves, T. L.; Varghese, J. N.; Drummond, C. J. Incorporation of the dopamine D2L receptor and bacteriorhodopsin within bicontinuous cubic lipid phases. 1. Relevance to in meso crystallization of integral membrane proteins in monoolein systems. Soft Matter 2010, 6, 48284837.

(23) Angelova, A.; Ollivon, M.; Campitelli, A.; Bourgaux, C. Lipid Cubic Phases as Stable Nanochannel Network Structures for Protein Biochip Development: X-ray Diffraction Study. Langmuir 2003, 19, 6928-6935.

(24) Barauskas, J.; Johnsson, M.; Joabsson, F.; Tiberg, F. Cubic Phase Nanoparticles (Cubosome): Principles for Controlling Size, Structure, and Stability. Langmuir 2005, 21, 2569-2577.

(25) Razumas, V. In Bicontinuous Liquid Crystals; Lynch, M. L., Spicer, P. T., Eds.; CRC Press: Boca Raton, FL, 2005; pp 169-212.

(26) Ericsson, B.; Larsson, K.; Fontell, K. A cubic protein-monooleinwater phase. Biochim. Biophys. Acta, Biomembr. 1983, 729, 23-27.

(27) Lendermann, J.; Winter, R. Interaction of cytochrome $c$ with cubic monoolein mesophases at limited hydration conditions: The effects of concentration, temperature and pressure. Phys. Chem. Chem. Phys. 2003, 5, 1440-1450.

(28) Lendermann, J.; Winter, R. Pressure-Induced Phase Changes of Cubic Monoolein - Cytochrome $c$ Mesophases. High Pressure Res. 2003, 23, 101-104.

(29) Kraineva, J.; Narayanan, R. A.; Kondrashkina, E.; Thiyagarajan, P.; Winter, R. Kinetics of Lamellar-to-Cubic and Intercubic Phase Transitions of Pure and Cytochrome $c$ Containing Monoolein Dispersions Monitored by Time-Resolved Small-Angle X-ray Diffraction. Langmuir 2005, 21, 3559-3571.

(30) Funari, S. S.; Rebbin, V.; Marzorati, L.; di Vitta, C. Membrane Morphology Modifications Induced by Hydroquinones. Langmuir 2011, 27, 8257-8262.

(31) Andreozzi, P.; Funari, S. S.; La Mesa, C.; Mariani, P.; Ortore, M. G.; Sinibaldi, R.; Spinozzi, F. Multi- to Unilamellar Transitions in Catanionic Vesicles. J. Phys. Chem. B 2010, 114, 8056-8060.

(32) Rappolt, M.; Di Gregorio, G. M.; Almgren, M.; Amenitsch, H.; Pabst, G.; Laggner, P.; Mariani, P. Non-equilibrium formation of the cubic Pn $3 m$ phase in a monoolein/water system. Europhys. Lett. 2006, $75,267-273$

(33) Chung, H.; Caffrey, M. The neutral area surface of the cubic mesophase: location and properties. Biophys. J. 1994, 66, 377-381.
(34) Pisani, M.; Bernstorff, S.; Ferrero, C.; Mariani, P. Pressure induced cubic-to-cubic phase transition in monoolein hydrated system. J. Phys. Chem. B 2001, 105, 3109-3119.

(35) Briggs, J.; Chung, H.; Caffrey, M. The TemperatureComposition Phase Diagram and Mesophase Structure Characterization of the Monoolein/Water System. J. Phys. II 1996, 6, 723-751.

(36) Turner, D. C.; Wang, Z.-G.; Gruner, S. M.; Mannock, D. A.; McElhaney, R. N. Structural study of the inverted cubic phases of didodecyl alkyl- $\beta$-D-glucopyranosyl-rac-glycerol. J. Phys. II 1992, 2, 2039-2063.

(37) Di Gregorio, G. M.; Mariani, P. Rigidity and spontaneous curvature of lipidic monolayers in the presence of trehalose: a measurement in the DOPE inverted hexagonal phase. Eur. Biophys. J. 2005, 34, 67-81.

(38) Israelachvili, N.; Mitchell, D.; Ninham, B. W. Theory of selfassembly of hydrocarbon amphiphiles into micelles and bilayers. J. Chem. Soc., Faraday Trans. 2 1976, 72, 1525-1568.

(39) Qiu, H.; Caffrey, M. The phase diagram of the monoolein/water system: metastability and equilibrium aspects. Biomaterials 2000, 21, 223-234.

(40) International Tables of X-ray Crystallography; The Kynoch Press: Birmingham, 1952. 